\title{
Respiratory Signal Extraction for 4D CT Imaging of the Thorax from Cone-Beam CT Projections
}

\author{
Simon Rit ${ }^{1}$, David Sarrut ${ }^{1,2}$, and Chantal Ginestet ${ }^{2}$ \\ 1 LIRIS, Université Lumière Lyon 2, 5 Av. P. Mendès-France, 69676 Bron, France \\ ${ }^{2}$ Radiotherapy Dpt, Centre Léon Bérard, 28 rue Laënnec, 69353 Lyon, France
}

\begin{abstract}
Current methods of four-dimensional (4D) CT imaging of the thorax synchronise the acquisition with a respiratory signal to restrospectively sort acquired data. The quality of the 4D images relies on an accurate description of the position of the thorax in the respiratory cycle by the respiratory signal. Most of the methods used an external device for acquiring the respiratory signal. We propose to extract it directly from thorax cone-beam (CB) CT projections. This study implied two main steps: the simulation of a set of CBCT projections, and the extraction, selection and integration of motion information from the simulation output to obtain the respiratory signal. A real respiratory signal was used for simulating the CB acquisition of a breathing patient. We extracted from CB images a respiratory signal with $96.4 \%$ linear correlation with the reference signal, but we showed that other measures of the quality of the extracted respiratory signal were required.
\end{abstract}

\section{Introduction}

Four-dimensional (4D) CT imaging, defined by Keall [1] as the "acquisition of a sequence of CT image sets over consecutive segments of a breathing cycle", constitutes the first step of $4 \mathrm{D}$ radiotherapy, which is "the explicit inclusion of the temporal changes in anatomy during the imaging, planning and delivery of radiotherapy". The 4D CT imaging of a free-breathing thorax must face one major technical problem: the time required ( $\simeq 0.5$ second per scan) for acquiring several complete $3 \mathrm{D}$ $\mathrm{CT}$ images along one respiratory cycle ( $\simeq 4$ second). Mori et al 2 have proposed a prototype 256-slice CT-scanner dedicated to real-time 4D imaging. Other methods currently in use address the slowness of actual $\mathrm{CT}$ acquisitions relatively to the respiratory cycle on both scanner geometries: spiral/helical and cone-beam (CB).

The respiratory correlated method is based on the spatial periodicity of respiration: suppose a respiratory signal $f(t)$, representing the periodic spatial variations of the position of the thorax in the respiratory cycle during acquisition; it is possible to sort a posteriori acquired data into $n$ bins and reconstruct $n$ 3D images 3 4 5. 5. Different respiratory synchronization techniques have been used: Vedam et al [3, Underberg et al [4] and Pan et al [5] synchronized CT acquisition with the position of the thorax skin surface in the antero-posterior direction; Low et al used a spirometer [6]; Damen et al [7] a thermometer under the patient's nose. 
Grangeat et al 8] also proposed a dynamic reconstruction algorithm with CB projections obtained from a CBCT system with a fast rotative gantry, without such synchronization signal. However, the gantry rotation of modern CBCT systems coupled with a linear accelerator is too slow to apply Grangeat's dynamic reconstruction.

With the respiratory correlated method, the critical issue stands with the quality of the respiratory signal, whatever the scanner geometry. Contradictory studies have explored the accuracy of these signals. Depending on the studies, the method using the antero-posterior position of the skin surface does not seem totally accurate [9] [10] whereas the air volume obtained with a spirometer seems to have better results [11. This is why some have proposed methods extracting the signal directly from image data, without any marker, both on spiral CT scan slices [5] and on CBCT projections [12].

We propose to analyze the motion of thorax CBCT projections obtained using a CBCT scan system with a slow rotating gantry to accurate respiratory signal extraction from image data. This study implied two main steps: the simulation of a set of CBCT acquisitions from a 4D model and a respiratory signal, and the extraction, selection and integration of motion information from the simulation output to obtain the respiratory signal for the time of acquisition. The simulation uses a reference respiratory signal, used as a gold standard for evaluating the results of our method.

\section{Cone-Beam CT Acquisition Simulation}

Our simulation follows a temporal respiratory signal; at each instant $t$, the respiratory signal (section 2.1) gives the position $f(t)$ in the respiratory cycle and the $4 \mathrm{D}$ model (section 2.2) gives the corresponding 3D volume. Digitally Reconstructed Radiographs (DRR) of the volume, i.e. CBCT projections, are then computed using a home made shearwarp algorithm [13.

\subsection{Respiratory Signal}

For each given time $t$, the respiratory signal indicates the position of the thorax in the respiratory cycle, denoted by $f: \mathbb{R} \rightarrow \mathbb{R}^{n}$. We define that $f$ must respect the following properties: when $f\left(t_{1}\right)=f\left(t_{2}\right)$, the thorax has the same spatial configuration at times $t_{1}$ and $t_{2}$; $\mathrm{f}$ is continuous, meaning that the spatial configuration at time $t+\varepsilon$ is almost equivalent to the one at time $t$ when $\varepsilon$ is very small. Previous studies 14 have suggested that the signal can be characterized in first approximation by a $1 \mathrm{D}$ function $(n=1)$. It is pseudo-periodic with a pseudo-period around 4 seconds and its extrema often correspond to maximal expiration and inspiration.

\subsection{D Model}

From $f(t)$, we must determine the corresponding spatial volume (spatial position) of the thorax, i.e. the corresponding 3D image. Our team had produced 
such a $4 \mathrm{D}$ model not elaborated from a complete $4 \mathrm{D}$ acquisition but from two $3 \mathrm{D}$ breathhold acquisitions, one at the end of normal expiration (I1) and one at the end of normal inspiration (I2), acquired using spiral CT imaging and the Active Breathing Coordinator (ABC, Elekta Oncology Systems) [15]. The non-rigid registration of I2 on I1 produced a dense vector-field representing the displacement of each point of I2 toward I1. From these displacements, and supposing in first approximation that each point of the thorax moves on a line, we could interpolate intermediate positions between the two extrema and produce a 4D image. We computed the air volume of the lungs by thresholding and morphological operations, indexing thus the different 3D images in the respiratory cycle and obtaining our 4D model.

\section{Respiratory Signal Extraction}

Visual observation of the motion of a set of CBCT projections, acquired from a free-breathing patient, intuitively led us to believe that the respiratory signal could be extracted a posteriori from this set of images. Following the same path, Zijp et al [12] have focused on the diaphragmatic cupola and projected their CB projections in the cranio-caudal direction, which produced a set of $1 \mathrm{D}$ signals from which they extracted the respiratory signal. The result is not compared to a reference for validation of its accuracy.

We propose here a study of the motion in a sequence of 2D CBCT projections for respiratory signal extraction. The method comports three sequential parts: selection of points of interest, motion extraction and trajectory processing.

\subsection{Points of Interest}

Some specific parts of the thorax are generally observed for motion extraction [14 12 10, like diaphragmatic cupolas or lung walls. Instead, we chose to consider a uniform set of points constituting a sub-sampling of the pixels of $\mathrm{CB}$ projections for a complete study of motion in sequential CBCT projections, with no limitation to any anatomic part. We typically used between 100000 and 200000 points of interest in total.

\subsection{Motion Extraction}

Our aim was to follow each point from $\mathrm{CB}$ projection to $\mathrm{CB}$ projection, i.e. the motion of points in the $2 \mathrm{D}$ projective space over the time of acquisition. We used a Block Matching Algorithm (BMA) 8].

CBCT imaging does not provide the projection of $3 \mathrm{D}$ points in a $2 \mathrm{D}$ space, but the integration of tissue densities on lines, which implies that the application of the BMA in the $2 \mathrm{D}$ projective space does not follow the projection of a $3 \mathrm{D}$ point, but the projection of a high tissue contrast 3D area, like chest and lung walls. It is then almost inevitable to lose the trajectory of the projected area after a significant rotation. 
We took this observation into account when using the BMA. Firstly, we computed the most probable trajectory of each point of interest by using the BMA from $\mathrm{CB}$ projection to $\mathrm{CB}$ projection, using the maximum linear correlation coefficient $C C$ as similarity measure. Secondly, we detected when the BMA is not following the original high contrast area projection: we compared each newly detected block to the original block and stopped when the $C C$ fell under a given user-defined threshold.

\subsection{Trajectory Processing}

Motion analysis of the set of points of interest produced a set of trajectories $E\{T\}$ where $T: \mathbb{N} \rightarrow \mathbb{N}^{2}$ defines the spatial position $(x, y)$ in the $2 \mathrm{D}$ projective space of a pixel, for CB projections $P_{i}$ with $0 \leq i<N$, and $N$ being the total number of CB images. These pieces of signal were processed to reconstruct the respiratory signal over the complete time of acquisition, respecting the characteristics of the respiratory signal described in 2.1 above.

Trajectory projection. We obtained a $1 \mathrm{D}$ respiratory signal by transforming the function $T: \mathbb{N} \rightarrow \mathbb{N}^{2}$ into a function $f: \mathbb{N} \rightarrow \mathbb{R}$, using a method that preserved maximum motion information, i.e. variations of the position of the point of interest over time. We firstly calculated the unit vector $\vec{u}$ corresponding to the best approximation line of all positions $M_{i}(x, y)$ with a linear regression and the mean $2 \mathrm{D}$ spatial position of points in time $C(\bar{x}, \bar{y})$. Then, for each projection $i$, the $1 \mathrm{D}$ projection value of the $2 \mathrm{D}$ spatial position $M_{i}(x, y)$ was given by $f_{T}(i)=\left\|\overrightarrow{C M}_{i} \cdot \vec{u}\right\|$ which is the norm of the projection of $\overrightarrow{C M}_{i}$ on the best-fit line (figure 11).
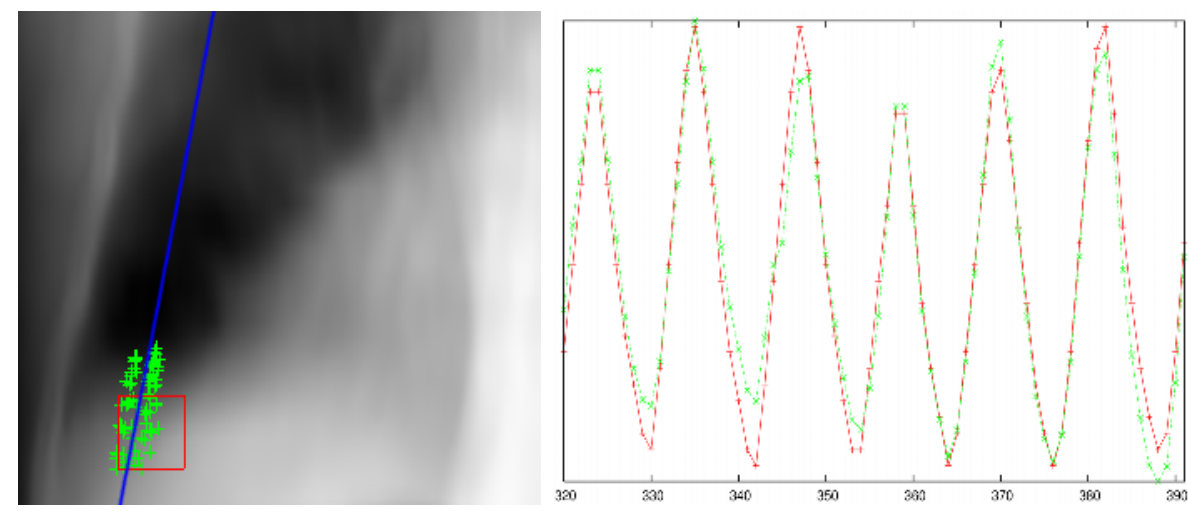

Fig. 1. On the left: the bottom left profile of a simulated CB projection of the lungs, with the selected trajectory (green) of an original block (red) and the best-fit line (blue). On the right: the computed 1D piece of respiratory signal (green) compared to the reference (red). The $\mathrm{X}$ axis represents the number of CB projections (chronologically numbered) and the $\mathrm{Y}$ axis the respiratory signal value $f(t)$. 


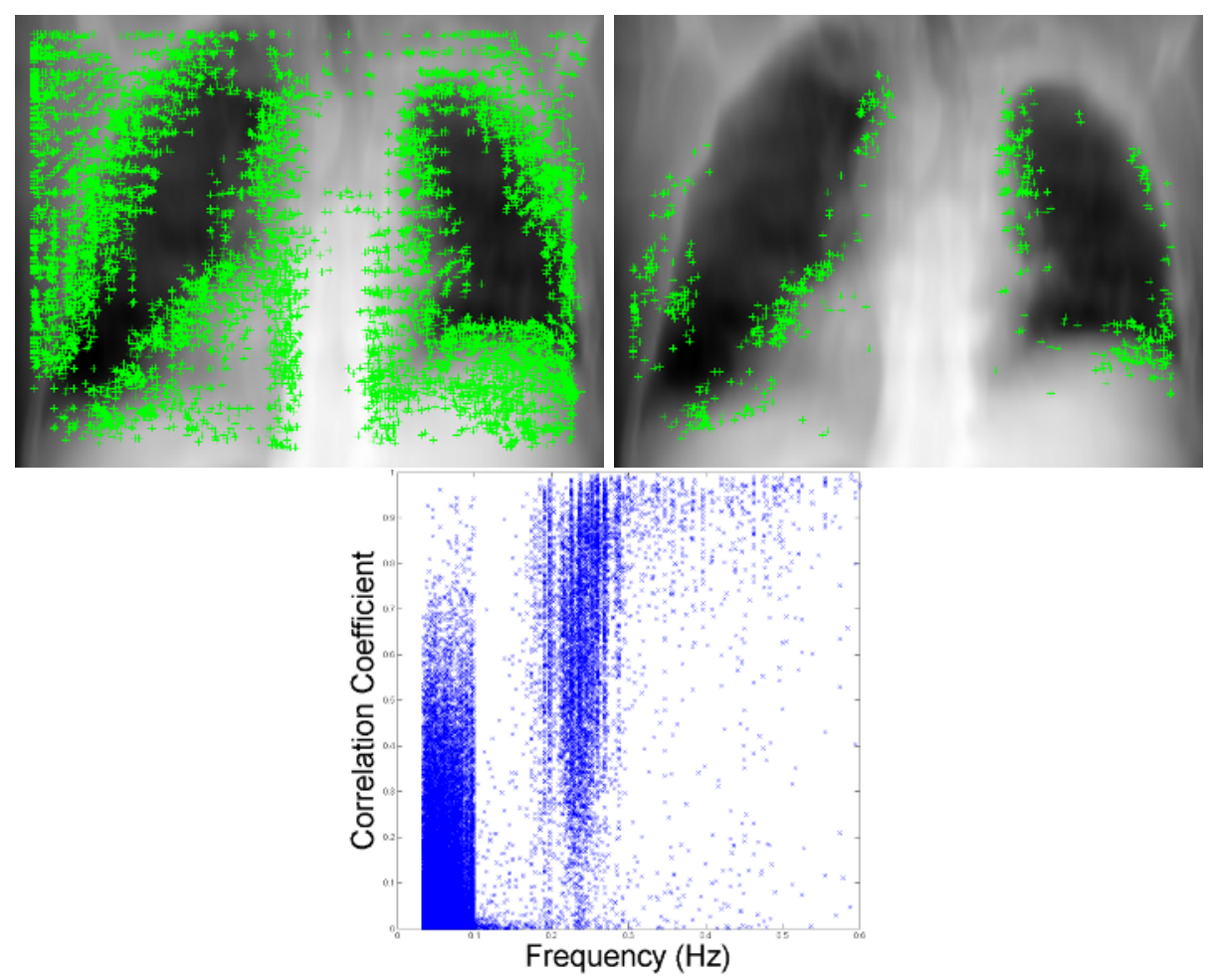

Fig. 2. On the left: a CBCT projection with points extracted by the BMA; on the right: the selected final points. In between: the point $\left(u_{\max }, C C\right)$ drawn for each piece of signal demonstrates the efficacy of frequency selection. Most of the signal pieces highly correlated to the reference have a period between 3 and $5 \sec (0.33$ and $0.2 \mathrm{~Hz})$.

Filtering. These 1D pieces of signal generally contain information about the respiratory signal, but this information is correlated with the motion due to cone-beam rotation. As this motion corresponds to an ellipsoidal motion with low frequency, we simply processed the data through a Fourier high pass filter.

Selection. Integration of the different pieces of signal provided good results, but an individual visual observation of the different trajectories showed that most were not relevant to the respiratory signal and a selection was required. We first eliminated short signals $(<7$ seconds) or signals with weak mean absolute amplitude $(<2$ pixels) because their spatial and/or temporal resolutions were not satisfactory.

To find an interesting selection parameter $p$, we refered to an important characteristic of the respiratory signal, its temporal pseudo-periodicity. We measure the higher amplitude value of the signal in the Fourier domain, i.e. $p=u_{\max }$ with $F\left(u_{\max }\right)=\max (|F(u)|), u \rightarrow|F(u)|$ being the Fourier amplitude spectrum. Selected signals have $p \simeq p_{\text {ref }}$, where $p_{\text {ref }}$ corresponds to the pseudo-period of respiration. 
This selection criterion was validated using the gold standard as reference by calculation of $C C$ for each piece of signal. After drawing $C C(p)$ (figure 2), we observed that $p$ for all signal pieces with high $C C$ approximated a particular value $p_{\text {ref }} \simeq 4 \mathrm{sec}$, which confirms our supposition.

Integration. Selected signals represent pieces of the respiratory signal over overlapping intervals of the time of acquisition. We integrated all the signals to determine the whole respiratory signal over the time of acquisition. We considered that extreme positions were matched for all thorax points, thus making integration simpler. Firstly, we linearly normalized all signal pieces between 0 and 1 and eventually put them in phase when they were in phase opposition. For each time $t$, the respiratory signal value was equal to the mean of the values of all different pieces at this time.

\section{Experiments and Results}

Experiments. We simulated $720 \mathrm{CB}$ projections, one every $0.5^{\circ}$ and every 0.36 second (Elekta Synergy parameter [12]), with a resolution of $600 \times 460$. Simulation was based on a real respiratory signal (irregular in phase and amplitude), acquired with an ABC system and two CT 3D breath-hold images of $512 \times 512 \times 65$ voxels. Motion extraction used blocks of 40x40 pixels and stopped when $C C$ fell under $93 \%$. We selected trajectories with a maximum period in the Fourier domain between 3 and 5 seconds ( 0.33 and $0.2 \mathrm{~Hz}$ ).

Measurement. The measure commonly used for evaluating respiratory signals with respect to a reference is the $C C$ [10][11. Here we also calculated another measure in direct relation with our use of the respiratory signal. We sorted both the respiratory signal extracted from CB images (result bins) and the gold standard (reference bins), then measured the percentage of misplaced samples in the result bins compared to the reference ones. We also calculated $\bar{\sigma}$, the average of $\sigma$, the respiratory signal standard deviation from reference values in each bin, for both reference and result bins, and their ratio $\bar{\sigma}_{\text {ref }} / \bar{\sigma}_{\text {res }}$. The smaller $\bar{\sigma}$ is, the more data in each bin were in phase with the respiratory cycle. $\bar{\sigma}_{r e f}$ automatically decreases with the number of bins, but $\bar{\sigma}_{r e s}$ stops decreasing when the number of bins is too big because of the inaccuracy of the respiratory signal result. $\bar{\sigma}_{\text {ref }} / \bar{\sigma}_{\text {res }}$ is equal to 1 when both sortings are equally good and decreases when the quality of the result sorting decreases.

Binning. There are different methods for sorting CB images with the respiratory signal, depending whether one takes hysteresis into account and whether bins have the same size. If bins have the same size, the 3D images have homogeneous resolutions but variation of the respiratory signal in each bin is variable; else, extrema have a higher resolution because there are more data used for their reconstruction. We chose to take hysteresis into account and to divide the signal into equal bins.

We extracted a respiratory signal with $96.4 \%$ correlation with the reference. After sorting, we obtain: 


\begin{tabular}{|c|c|c|c|c|}
\hline Number of bins & Misplaced samples & $\bar{\sigma}_{\text {ref }}$ & $\bar{\sigma}_{\text {res }}$ & $\bar{\sigma}_{\text {ref }} / \bar{\sigma}_{\text {res }}$ \\
\hline 1 & 0 & 0.249 & 0.249 & 1 \\
2 & $23(3 \%)$ & 0.123 & 0.127 & 0.97 \\
4 & $57(8 \%)$ & 0.093 & 0.101 & 0.92 \\
8 & $122(17 \%)$ & 0.055 & 0.068 & 0.81 \\
12 & $184(25 \%)$ & 0.038 & 0.055 & 0.69 \\
\hline
\end{tabular}

\section{Discussion and Conclusion}

An interesting result of this method is the location of selected trajectories on CB images, i.e. points of CBCT images having a motion directly related to respiration. Most of these points are located around the diaphragmatic cupola, which corroborates Zijp et al [12] hypothesis, but others are also located on lung walls in the inferior lobe (figure 2). Their motion direction depends on their location: mostly cranio-caudal for points around the cupola, and perpendicular to the lung walls otherwise. The location of the points of interest has now been determined; future work will improve the method with a priori detection of the points of interest.

The computed respiratory signal is visually very close to the reference, as confirmed by the high $C C$. But the result bins has a proportion of misplaced points compared to the reference bins which increases with the number of bins. The impact of these misplacements is measured by $\bar{\sigma}_{r e f} / \bar{\sigma}_{r e s}$. It is unnecessary to increase the number of bins if $\bar{\sigma}_{r e f} / \bar{\sigma}_{\text {res }}$ is too low, and/or if it does not decrease sufficiently $\bar{\sigma}$. This measurement points out the importance of an accurate respiratory signal for $4 \mathrm{D}$ imaging, and the not so good capacity for the $C C$ to measure this accuracy. However, the number of bins is limited because the quantity of data in each bin is a primordial criterion for the quality of the reconstruction.

4D CT images will be reconstructed with a number of bins deduced from these observations. They will be used for modeling the thorax motion, and will offer the possibility to take motion into account for treatment planning. Future work will also include validation on real CBCT data.

Acknowledgement. This work was supported in part by Elekta Oncology Systems.

\section{References}

1. Keall, P.: 4-dimensional computed tomography imaging and treatment planning. Radiation Oncology 14 (2004) 80-90

2. Mori, S., Endo, M., Tsunoo, T., Kandatsu, S., Tanada, S., Aradate, H., Saito, Y., Miyazaki, H., Satoh, K., Matsushita, S., Kusakabe, M.: Physical performance evaluation of a 256-slice CT-scanner for four-dimensional imaging. Medical Physics 31 (2004) 1348-1356 
3. Vedam, S., Keall, P., Kini, V., Mostafavi, H., Shukla, H., Mohan, R.: Acquiring a four-dimensional computed tomography dataset using an external respiratory signal. Physics in Medicine and Biology 48 (2003) 45-62

4. Underberg, R.W., Lagerwaard, F.J., Cuijpers, J.P., Slotman, B.J., van Sörnsen de Koste, J.T., Senan, S.: Four-dimensional CT scans for treatment planning in stereotactic radiotherapy for stage I lung cancer. International Journal of Radiation Oncology Biology Physics 60 (2004) 1283-1290

5. Pan, T., Lee, T.Y., Rietzel, E., Chen, G.T.Y.: 4D-CT imaging of a volume influenced by respiratory motion on multi-slice CT. Medical Physics 31 (2004) 333-341

6. Low, D.A., Nystrom, M., Kalinin, E., Parikh, P., Dempsey, J.F., Bradley, J.D., Mutic, S., Wahab, S.H., Islam, T., Christensen, G., Politte, D.G., Whiting, B.R.: A method for the reconstruction of four-dimensional synchronized CT scans acquired during free breathing. Medical Physics 30 (2003) 1254-1263

7. Damen, E., Wolthaus, J., Herk, M.V., Sonke, J.J., Remeijer, P., Zijp, L.: Imageguided radiotherapy for lung cancer: Respiration correlated cone-beam CT to verify tumor position and motion characteristics during treatment delivery (abstract). In: ASTRO. (2004)

8. Grangeat, P., Koenig, A., Rodet, T., Bonnet, S.: Theoretical framework for a dynamic cone-beam reconstruction algorithm based on a dynamic particle model. Physics in Medicine and Biology 47 (2002) 2611-2625

9. Ford, E., Mageras, G., Yorke, E., Ling, C.: Respiration-correlated spiral CT: A method of measuring respiratory-induced anatomic motion for radiation treatment planning. Medical Physics 30 (2003) 88-97

10. Koch, N., Liu, H.H., Starkschall, G., Jacobson, M., Forster, K., Liao, Z., Komaki, R., Stevens, C.W.: Evaluation of internal lung motion for respiratory-gated radiotherapy using MRI: Part I-correlating internal lung motion with skin fiducial motion. Int Journal of Radiation Oncology Biology Physics 60 (2004) 1459

11. Lu, W., Parikh, P.J., Naqa, I.M.E., Nystrom, M.M., Hubenschmidt, J.P., Wahab, S.H., Mutic, S., Singh, A.K., Christensen, G.E., Bradley, J.D., Low, D.A.: Quantitation of the reconstruction quality of a four-dimensional computed tomography process for lung cancer patients. Medical Physics 32 (2005) 835-1228

12. Zijp, L., Sonke, J.J., Herk, M.V.: Extraction of the respiratory signal from sequential thorax cone-beam X-ray images. International Conference on the Use of Computers in Radiation Therapy (2004)

13. Lacroute, P.G.: Fast volume rendering using a shear-warp factorization of the viewing transformation. PhD thesis, Stanford University (1995)

14. Kondo, T., Kobayashi, I., Taguchi, Y., Ohta, Y., Yanagimachi, N.: A dynamic analysis of chest wall motions with MRI in healthy young subjects. Respirology $\mathbf{5}$ (2000) 19

15. Boldea, V., Sarrut, D., Clippe, S.: Lung deformation estimation with non-rigid registration for radiotherapy treatment. In: Medical Image Computing and ComputerAssisted Intervention MICCAI'2003. Volume 2878. (2003) 770-7 